\title{
Consensus-Based Distributed Optimization of Generation Dispatch of Multi-Area AC Systems Interconnected by DC Lines
}

\author{
Fan $\mathrm{ZHANG}^{\mathrm{a}}$, Hui JIANG ${ }^{\mathrm{b}, 1}$, Minghuan $\mathrm{WU}^{\mathrm{a}}$ and Jianchun PENG ${ }^{\mathrm{a}}$ \\ ${ }^{a}$ College of Mechatronics and Control Engineering, Shenzhen University, Shenzhen, \\ China \\ ${ }^{\mathrm{b}}$ College of Physics and Optoelectronic Engineering, Shenzhen University, Shenzhen, \\ China
}

\begin{abstract}
This paper is dedicated to solving the distributed optimization of generation dispatch of multi-area AC systems interconnected by DC lines, which aims at minimizing the total generation cost while satisfying the power supply demand balance and generation capacity constraints. A novel nodal loss formula which derived from the branch active power flow equation is proposed based on phase angle and impedance to improve the system economy. A distributed algorithm based on consensus is built to solve the generation dispatch problem. It has a great effect on improving convergence effect and rate of the system. The control strategy is used on the structure of multi-area interconnection, which improves the reliability of power supply and guarantee the power quality. The study was conducted using three area AC systems interconnected by DC lines. The simulation results show that the proposed generation dispatch method is reliable in convergence. It provides an effective tool for distributed optimization of generation dispatch of multi-area AC systems interconnected by DC lines.
\end{abstract}

Keywords. Multi-area AC system, DC lines, multi-area interconnection

\section{Introduction}

Generation dispatch can improve the operation efficiency and income of power system, which is the basis of optimal operation of power grid. Moreover, it can save energy and is of great value to the rational allocation and adjustment of power resource structure $[1,2]$. The essence of power system generation dispatch is to meet the load demand of users under the condition of power balance and security constraints, and to achieve the optimal generation benefit of power system by reasonably arranging the operation mode of generators [3].

In recent years, researchers at home and abroad have carried out a lot of research on the scheduling optimization of multi-area interconnected power grid. The reliability and quality of power supply of a single small power grid cannot be guaranteed. Multiarea $\mathrm{AC}$ systems interconnection is conducive to improving power quality, performing generation dispatch in a wider range and obtaining greater economic benefits. What's

${ }^{1}$ Corresponding Author, Hui JIANG, College of Physics and Optoelectronic Engineering, Shenzhen University, Shenzhen, China; Email: huijiang@szu.edu.cn; 1910294034@email.szu.edu.cn. 
more, it can provide power supply and reserve for each other among areas, enhance the ability to resist accidents, and improve the security level and power supply reliability of power grid [4-6].

For large scale grids, especially the interconnected multi-area systems, it is difficult for the central-controlled strategies to satisfy the demand of social power consumption, and it's prone to information leakage [7, 8]. For the distributed generation, each node not only has the information of its own and has easy access to information about its neighbours. This way of power generation greatly improves the communication ability and information confidentiality [9]. Even if there is a single point of failure, it still works efficiently, in line with the trend of power system development [10].

On the basis of consensus theory, this paper studies distributed optimization for generation dispatch of multi-area AC systems interconnected by DC lines, aiming at minimizing the total generation cost. Firstly, taking the minimum cost of all generators as the objective function and combining the constraints of the power balance and generator outputs, the distributed-control optimal generation dispatch model of grid is established. Secondly, a novel bus loss formula is proposed, which derived from the branch active power flow equation. According to the bus loss, we get the node power balance equation, and then derive the update formula of phase angle and DC line power. Thirdly, based on the consistency algorithm, the calculation formula of incremental cost is derived. Derivation of Lagrange function is obtained to calculate the power of generator. Finally, a simulation case is designed to test the proposed control method. The simulation results show that the proposed method can minimize the operation cost of multi-area AC systems interconnected by DC lines.

\section{Generation Dispatch Model}

\subsection{Objective Function}

The objective function is to minimize the cost of fuel and emission. In this way, it becomes a multi-objective optimization problem. Therefore, this paper uses the weighting method to solve the problem [11].

$$
\min : F=\sum_{i=1}^{n} C_{i}\left(P_{G i}\right)+\delta \sum_{i=1}^{n} E_{i}\left(P_{G i}\right)
$$

where F represents the total cost of the model; $C_{i}\left(P_{G i}\right)$ is the fuel cost of unit $i ; E_{i}\left(P_{G i}\right)$ is the emission cost of unit $i ; \delta$ represents the weighting factor.

(1) Fuel cost function

$$
C_{i}\left(P_{G i}\right)=a_{i} P_{G i}^{2}+b_{i} P_{G i}+c_{i}
$$

where $a_{i}, b_{i}, c_{i}$ represent the correlation coefficients of fuel cost of unit $i$.

(2) Emission cost function

$$
E_{i}\left(P_{G i}\right)=d_{i} P_{G i}^{2}+e_{i} P_{G i}+f_{i}
$$


where $d_{i}, e_{i}, f_{i}$ represent the correlation coefficients of emission cost of unit $i$.

\subsection{Novel Node Loss Formula and Node MW Balance Equations}

For branch $(i, j)$, if parallel branch is ignored, the active power flow equation of branch can be expressed by equation (4):

$$
P_{i j}=\left(U_{i j}^{2}-U_{i} U_{j} \cos \theta_{i j}\right) g_{i j}-U_{i} U_{j} \sin \theta_{i j} b_{i j}
$$

where $g_{\mathrm{ij}}$ is the branch conductance, $\mathrm{b}_{\mathrm{ij}}$ is the branch susceptance.

The power system has the following characteristics: in per unit system, the node voltage tends to the rated voltage, the amplitude is close to 1 (the deviation is in $\pm 5 \%$ ), and the phase angle difference $\theta_{i j}$ between the two ends of each branch is very small and close to 0 , and the line resistance is far less than the reactance [12]. Therefore, the approximation is rational: $U_{i}=U_{j}=1, \sin \theta_{i j}=\theta_{i j}, \cos \theta_{i j}=1, r_{i j}=0$. As a result, (4) can be simplified as (5):

$$
P_{i j}=-b_{i j}\left(\theta_{i}-\theta_{j}\right)=\left(\theta_{i}-\theta_{j}\right) / x_{i j}
$$

where $b_{i j}=-1 / x_{i j}, x_{i j}$ is the branch reactance.

Similar to Ohm's law of DC circuit, taking $P_{i j}$ as DC current, $\theta_{i}$ and $\theta_{j}$ as node $i$ voltage and node $j$ voltage, $x_{i j}$ as the branch resistance, the nonlinear active power flow equation can be simplified as the following linear equation-the DC power flow equation (5).

In order to formulate node loss, we replacing $x_{i j}$ in (5) with line impedance modulus as follows.

$$
w_{i j}=\left(r_{i j}^{2}+x_{i j}^{2}\right)^{0.5}
$$

The transmission loss on branch $i-j$ can then be described as follows.

$$
P_{i j-l o s s}=\left(\frac{\theta_{i}-\theta_{j}}{w_{i j}}\right)^{2} r_{i j}
$$

Allocating equally each line loss to its two terminals and taking $P_{i j}$ as DC current, we formulate the node $i$ loss as follows.

$$
P_{i l o s s}=0.5 \sum_{j \in i}\left(\frac{\theta_{i}-\theta_{j}}{w_{i j}}\right)^{2} r_{i j}
$$

Counting node loss, we have the node MW balance equations as follows for common node and tie-line node, respectively. 


$$
\left\{\begin{array}{l}
P_{G i}-P_{D i}=\sum_{k \in i} \frac{\theta_{i}-\theta_{k}}{x_{i k}}+0.5 \sum_{k \in i}\left(\frac{\theta_{i}-\theta_{k}}{w_{i k}}\right)^{2} r_{i k} \\
P_{G j}-P_{D j}-P_{\text {line }}=\sum_{k \in i} \frac{\theta_{j}-\theta_{k}}{x_{j k}}+0.5 \sum_{k \in i}\left(\frac{\theta_{j}-\theta_{k}}{w_{j k}}\right)^{2} * r_{j k}+0.5 * \beta * P_{\text {line }}
\end{array}\right.
$$

where $\beta$ is the loss rate of the tie-line ( $\mathrm{AC} / \mathrm{DC}$ converter $+\mathrm{DC}$ line $+\mathrm{DC} / \mathrm{AC}$ converter), and $3 \%$ is recommended here.

Manipulating (9), we have the following updating formulas for phase angle and tie-line power.

$$
\left\{\begin{array}{l}
\theta_{i}=\frac{P_{G i}-P_{D i}+\sum_{k \in i} \frac{\theta_{k}}{x_{i k}}-0.5 * \sum_{k \in i}\left(\frac{\theta_{k}}{w_{i k}}\right)^{2} r_{i k}}{\sum_{k \in i} \frac{1}{x_{i k}}+0.5 * \theta_{i} \sum_{k \in i} \frac{r_{i k}}{w_{i k}^{2}}-\sum_{k \in i} \frac{\theta_{k}}{w_{i k}^{2}} r_{i k}} \\
\theta_{j}=\frac{P_{G j}-P_{D j}-P_{\text {line }}+\sum_{k \in j} \frac{\theta_{k}}{x_{j k}}-0.5 * \sum_{k \in j}\left(\frac{\theta_{k}}{w_{j k}}\right)^{2} r_{j k}-\frac{1}{2} * \beta * P_{\text {line }}}{\sum_{k \in j} \frac{1}{x_{j k}}+0.5 * \theta_{j} \sum_{k \in j} \frac{r_{j k}}{w_{j k}^{2}}-\sum_{k \in j} \frac{\theta_{k}}{w_{j k}^{2}} r_{j k}} \\
P_{\text {line-i }}=\frac{P_{D i}-P_{G i}+\sum_{k \in i} \frac{\theta_{i}-\theta_{k}}{x_{i k}}+0.5 \sum_{k \in i}\left(\frac{\theta_{i}-\theta_{k}}{w_{i k}}\right)^{2} * r_{i k}}{d i r-0.5 * \beta}
\end{array}\right.
$$

Both terminals ( $i$ and $j$ ) of a tie-line have impact on tie-line power. So we use the average value of $P_{\text {line- } i}$ and $P_{\text {line-j }}$ as the tie-line power.

$$
P_{\text {line }}=\left(P_{\text {line-i }}+P_{\text {line-j }}\right) / 2
$$

\subsection{Consistency Algorithm}

According to the equal incremental cost theory, the incremental cost of slack node generator, can be determined by (12).

$$
\lambda_{s}=\lambda_{s}+2 a_{s} * \Delta P_{s}
$$

where $\Delta P_{s}$ is the deficit generation MW determined by (9).

$$
\Delta P_{s}=P_{D s}+P_{\mathrm{sj}}+P_{\text {sloss }}-P_{G s}
$$


The incremental cost of the other nodes, $\lambda_{i}$, are determined by consensus-method as follows.

$$
\lambda_{i}=\frac{1}{d_{i i}}\left(\sum_{j \in i} \alpha_{i j} \bullet \lambda_{j}[k]\right)
$$

where $d_{i i}$ is the number of edges associated with node $i, \alpha_{i j}$ refers to the weight of edges between node $i$ and $j ; d_{i i}$ and $\alpha_{i j}$ are elements of the Laplacian matrix in consensus method.

The generation power of the nodes, $P_{G i}$, are determined by the equal incremental cost method as follows. If a generation power exceeds its generator's upper (lower) limit, the generation power will be fixed at its upper (lower) limit.

$$
P_{G i}=\left\{\begin{array}{cc}
P_{G i \min } & P_{G i}<P_{G i \min } \\
\frac{\lambda_{i}-b_{i}-\delta e_{i}}{2 a_{i}+2 \delta d_{i}} & P_{G i \min }<P_{G i}<P_{G i \max } \\
P_{G i \max } & P_{G i}>P_{G i \max }
\end{array}\right.
$$

Transforming (10)-(15) to discrete form, we have the consistency algorithm formulas--dynamic updating formula, for phase angle, tie-line power, incremental cost and generator powers.

$$
\left\{\begin{array}{l}
\theta_{i}[k+1]=\varepsilon \theta_{i}[k]+(1-\varepsilon) \frac{P_{G i}-P_{D i}+\sum_{k \in i} \frac{\theta_{k}}{x_{i k}}-0.5 * \sum_{k \in i}\left(\frac{\theta_{k}}{w_{i k}}\right)^{2} r_{i k}}{\sum_{k \in i} \frac{1}{x_{i k}}+0.5 * \theta_{i} \sum_{k \in i} \frac{r_{i k}}{w_{i k}^{2}}-\sum_{k \in i} \frac{\theta_{k}}{w_{i k}^{2}} r_{i k}} \\
\theta_{j}[k+1]=\varepsilon \theta_{j}[k]+(1-\varepsilon) \frac{P_{G j}-P_{D j}-P_{\text {line }}+\sum_{k \in j} \frac{\theta_{k}}{x_{j k}}-0.5 * \sum_{k \in j}\left(\frac{\theta_{k}}{w_{j k}}\right)^{2} r_{j k}-\frac{1}{2} * \beta * P_{\text {line }}}{\sum_{k \in j} \frac{1}{x_{j k}}+0.5^{*} \theta_{j} \sum_{k \in j} \frac{r_{j k}}{w_{j k}^{2}}-\sum_{k \in j} \frac{\theta_{k}}{w_{j k}^{2}} r_{j k}} \\
P_{\text {linei }}[k+1]=\varepsilon P_{\text {linei }}[k]+(1-\varepsilon) \frac{P_{D i}-P_{G i}+\sum_{k \in i} \frac{\theta_{i}-\theta_{k}}{x_{i k}}+0.5 \sum_{k \in i}\left(\frac{\theta_{i}-\theta_{k}}{w_{i k}}\right)^{2} * r_{i k}}{d i r-0.5 * \beta} \\
\lambda_{s}[k+1]=\lambda_{s}[k]+2 a_{s} * \Delta P_{s} \\
\lambda_{i}[k+1]=\varepsilon \lambda_{i}[k]+(1-\varepsilon) \frac{1}{d_{i i}}\left(\sum_{j \in i} \alpha_{i j} \bullet \lambda_{j}[k]\right) \\
P_{G i}[k+1]=\varepsilon P_{G i}[k]+(1-\varepsilon) \frac{\lambda_{i}[k]-b_{i}-\delta e_{i}}{2 a_{i}+2 \delta d_{i}}
\end{array}\right.
$$

where $\varepsilon$ is the feedback gain coefficient. 


\section{Case Study}

\subsection{Structure of Three Area Interconnected Power System}

This study is carried on the three regions interconnected AC power system with three DC lines as shown in figure 1 to test the proposed approach. At the communication level, the three regions remain relatively independent and do not affect each other.

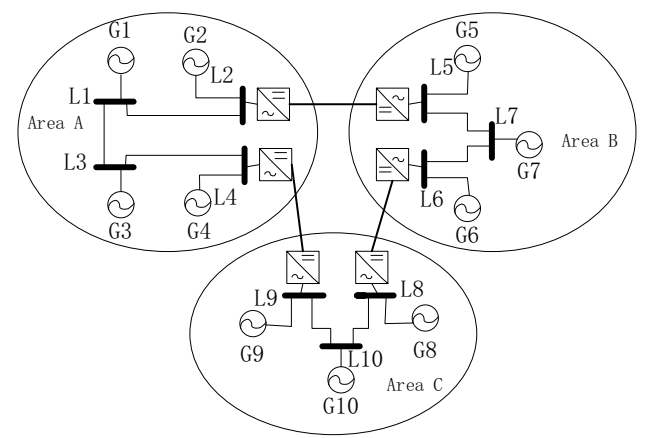

Figure 1. The structure of multi-area AC systems interconnected by DC lines.

\subsection{Simulation and Analysis}

Case: The simulation is carried out according to the above model and control strategy. The node parameters of multi-area AC systems interconnected by DC lines are shown in table 1. Figure 2 shows the iterative process of the consistency variables of each node in the three regions. The incremental generation costs of three regions are $\lambda_{1}=7.5206 \$ \mathrm{MWh}, \lambda_{2}=7.8290 \$ / \mathrm{MWh}, \lambda_{3}=7.6296 \$ / \mathrm{MWh}$. Figure 3 shows the iterative process of each generator. After 300 times iteration, $\mathrm{P}_{\mathrm{G} 1}=61.2446 \mathrm{MW}$, $\mathrm{P}_{\mathrm{G} 2}=103.8434 \mathrm{MW}, \mathrm{P}_{\mathrm{G} 3}=96.1331 \mathrm{MW}, \mathrm{P}_{\mathrm{G} 4}=88.3452 \mathrm{MW}, \mathrm{P}_{\mathrm{G} 5}=79.0661 \mathrm{MW}$, $\mathrm{P}_{\mathrm{G} 6}=103.1818 \mathrm{MW}, \mathrm{P}_{\mathrm{G} 7}=114.6231 \mathrm{MW}, \mathrm{P}_{\mathrm{G} 8}=65.8219 \mathrm{MW}, \mathrm{P}_{\mathrm{G} 9}=88.99565 \mathrm{MW}$, $\mathrm{P}_{\mathrm{G} 10}=101.0359 \mathrm{MW}$. Figure 4 shows the variation process of power deviation of each node, finally, they all converge to 0 . Figure 5 shows the transmission power variation process of three DC lines. The power of the DC lines is $\mathrm{P}_{\text {linel }}=24.8489 \mathrm{MW}, \mathrm{P}_{\text {line } 2}=23.9118 \mathrm{MW}, \mathrm{P}_{\text {line3 }}=20.9359 \mathrm{MW}$. The total generation cost is $6626.00 \$ / \mathrm{h}$.

Table 1. Node parameters of multi-area AC systems interconnected by DC lines.

\begin{tabular}{cccccccccc}
\hline & $a_{i} * 10^{\wedge}-3$ & $b_{i}$ & $c_{i}$ & $d_{i}$ & $e_{i}$ & $f_{i}$ & $P_{G i \max }$ & $P_{G i \min }$ & $P_{D i}$ \\
\hline $\mathbf{1}$ & 6.3 & 6.5 & 30 & 0.0041 & 0.3276 & 13.859 & 100 & 10 & 75 \\
$\mathbf{2}$ & 6.1 & 5.9 & 25 & 0.0041 & 0.3276 & 13.859 & 150 & 10 & 75 \\
$\mathbf{3}$ & 6.2 & 6.1 & 25 & 0.0068 & -0.5455 & 39.2669 & 100 & 10 & 75 \\
$\mathbf{4}$ & 6.7 & 6.14 & 16 & 0.0068 & -0.5455 & 39.2669 & 180 & 10 & 75 \\
$\mathbf{5}$ & 6.3 & 6.54 & 16 & 0.0041 & 0.3276 & 13.859 & 150 & 10 & 100 \\
$\mathbf{6}$ & 5.8 & 6.28 & 54 & 0.0041 & 0.3276 & 13.859 & 150 & 10 & 100 \\
$\mathbf{7}$ & 5.3 & 6.31 & 23 & 0.0068 & -0.5455 & 39.2669 & 150 & 10 & 100 \\
$\mathbf{8}$ & 6.3 & 6.54 & 16 & 0.0041 & 0.3276 & 13.859 & 150 & 10 & 100 \\
$\mathbf{9}$ & 5.8 & 6.28 & 54 & 0.0041 & 0.3276 & 13.859 & 150 & 10 & 100 \\
$\mathbf{1 0}$ & 5.3 & 6.31 & 23 & 0.0068 & -0.5455 & 39.2669 & 150 & 10 & 100 \\
\hline
\end{tabular}

Note: $P_{D i}$ is the power of the load. 


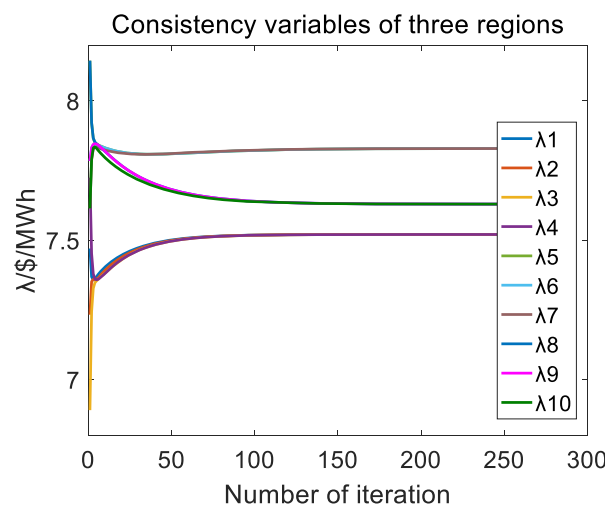

Figure 2. Consistency variables of three regions.

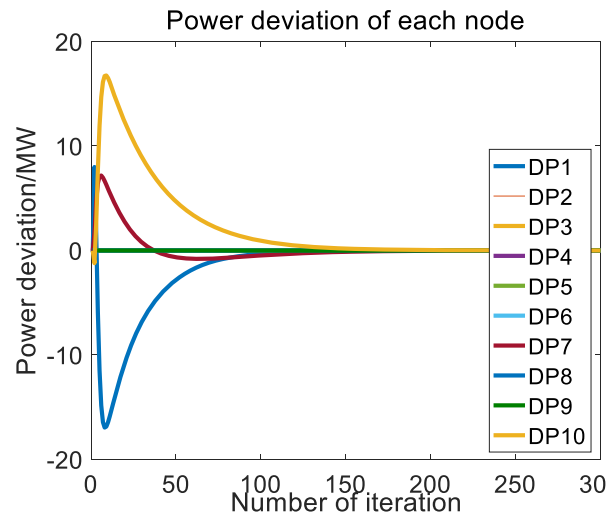

Figure 4. Power deviation of each node.

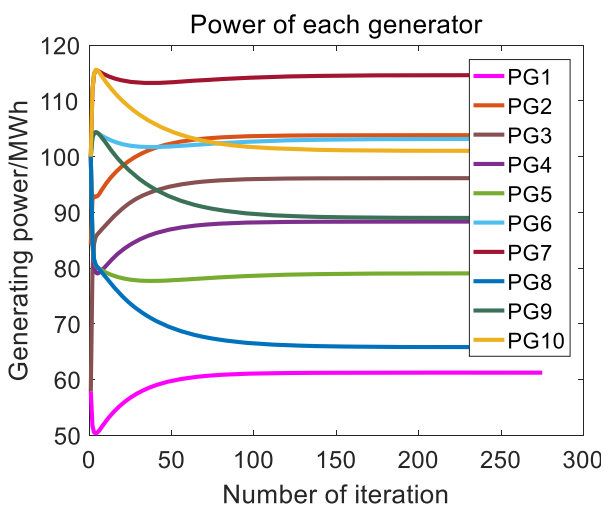

Figure 3. Power of each generator.

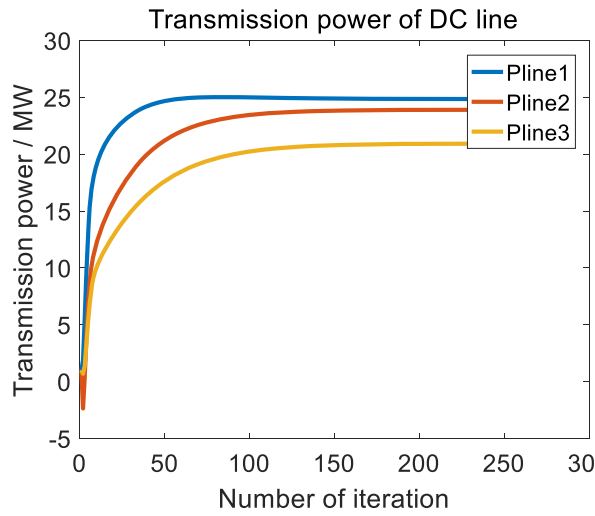

Figure 5. Transmission power of DC line.

\section{Conclusion}

This primary aim of the work was to proposed an advanced method for generation dispatch of multi-area AC systems interconnected by DC lines. It is found that using the consensus-based distributed optimization algorithm as a tool for solving the problem has a great effect on improving convergence effect and rate of the system. The control strategy is used on the structure of multi-area interconnection, which improves the reliability of power supply and guarantee the power quality. Finally, a novel approximate loss estimation method based on phase angle and impedance is proposed, it improves the system economy. The study was conducted using three area $\mathrm{AC}$ systems interconnected by DC lines. Results of the simulation shows the convergence effect of the system can be optimized. Therefore, the proposed approach can minimize the operating cost of the system and reduce the exhaust emission to achieve energy saving and environmental protection. 


\section{Acknowledgement}

This work was supported by Shenzhen Science and Technology Innovation Committee partly under Grant JCYJ20180305125407996, partly under Grant GJHZ20180928160212241, and partly under Grant JCYJ20190808165201648.

\section{References}

[1] Yang S, Tan S and Xu J 2013 Consensus Based Approach for Economic Dispatch Problem in a smart grid IEEE Transactions on Power Systems 28 4416-4426.

[2] Zhang Z and Chow M 2012 Convergence Analysis of the Incremental Cost Consensus Algorithm Under Different Communication Network Topologies in a Smart Grid. IEEE Transactions on Power Systems 27 1761-1768.

[3] Tang Z, Hill D J and Liu T 2018 A Novel Consensus-Based Economic Dispatch for Microgrids IEEE Transactions on Smart Grid 9 3920-3922.

[4] Sharma M, Pandit M and Srivastava L 2010 Multi-area economic dispatch with tie-line constraints employing evolutionary approach International Journal of Engineering, Science and Technology 2 141-143.

[5] Lin W M and Chen S J 2002 Bid-based dynamic economic dispatch with an efficient interior point algorithm International Journal of Electrical Power \& Energy Systems 24 51-57.

[6] Lu H Y, Sriyanyong P, Song Y H, et al. 2010 Experimental study of a new hybrid PSO with mutation for economic dispatch with non-smooth cost function International Journal of Electrical Power and Energy Systems 32 921-935.

[7] Olfati-Saber R, Fax J A and Murray R M 2007 Consensus and cooperation in networked multi-agent systems Proceedings of the IEEE 95 (1) 215-233.

[8] Zhao C, He J, Cheng P, et al. 2017 Analysis of consensus-based distributed economic dispatch under stealthy attacks IEEE Transactions on Industrial Electronics 64 5107-5117.

[9] Duan J and Chow M A 2019 Novel data integrity attack on consensus-based distributed energy management algorithm using local information IEEE Transactions on Industrial Informatics 15 15441553.

[10] Huang B, Li Y, Zhang H, et al. 2016 Distributed optimal co-multi-microgrids energy management for energy internet IEEE/CAA Journal of Automatica Sinica 3 357-364.

[11] Zhao H., Xu S, Yuan D, et al. 2011 Minimum communication cost consensus in multi-agent systems with Markov chain patterns IET Control Theory \& Applications 5 63-68.

[12] Zhao T, Li Z and Ding Z 2019 Consensus-based distributed optimal energy management with less communication in a microgrid IEEE Transactions on Industrial Informatics 15 3356-3367. 